Pathologe 2010 · 31:410-411

DOI 10.1007/s00292-010-1350-x

Online publiziert: 15. September 2010

(c) Springer-Verlag 2010

\author{
A. Hartmann ${ }^{1}$ - J. Rüschoff ${ }^{2}$ \\ ${ }^{1}$ Institut für Pathologie, Universität Erlangen \\ 2 Pathologie Nordhessen, Kassel
}

\title{
Pathologie familiärer Tumorsyndrome
}

\section{Prädiktive genetische Diagnostik}

Etwa 10\% aller Krebserkrankungen sind auf eine erbliche Tumordisposition zurückzuführen. Die molekularen Grundlagen der Disposition für eine Reihe autosomal-dominanter erblicher Tumorkrankheiten sind weitgehend aufgeklärt. Die Tatsache des dominanten Erbgangs und neue diagnostische Möglichkeiten können grundsätzlich für die Krebsprävention genutzt werden, da die Nachkommen eines solchen Patienten als Personen mit erhöhtem Risiko anzusehen sind.

Die Identifikation einer Keimbahnmutation bei einem Patienten eröffnet die Möglichkeit einer prädiktiven genetischen Diagnostik bei den gesunden Verwandten. So bedeutet der Ausschluss der in einer Familie nachgewiesenen und für eine Krebsdisposition verantwortlichen Mutation bei einem Angehörigen eine große Entlastung. Wird die betreffende Keimbahnmutation bei einem noch gesunden Verwandten nachgewiesen, kann diese Person in ein gezieltes Krebsfrüherkennungs- und -vorsorgeprogramm aufgenommen werden, damit bei Auftreten eines Tumors rechtzeitig medizinisch interveniert werden kann. Bei einigen Tumorerkrankungen kann mit prophylaktisch-chirurgischen Maßnahmen die Manifestation der Erkrankung weitgehend verhindert werden.

Die neuen Möglichkeiten der prädiktiven genetischen Diagnostik stellen für die Medizin und die Gesellschaft ganz neue Anforderungen dar, da bei jeder der in Betracht kommenden Krebsdispositionen mehrere medizinische Disziplinen koope- rieren sowie ethische, soziale und psychologische Aspekte besonders berücksichtigt werden müssen. Jede prädiktive Diagnostik muss mit großer Umsicht und Verantwortung durchgeführt werden. Die gesetzlichen Grundlagen dieser Diagnostik sind im Gendiagnostikgesetz niedergelegt worden, das im Jahr 2009 verabschiedet wurde.

Erbliche Krebsformen stellen keine monogenen Erkrankungen im eigentlichen Wortsinn dar. Vielmehr wird eine bestimmte genetische Läsion über die Keimbahn an alle Zellen weitergegeben und kann zu einer vorzeitigen Entwicklung von Neoplasien führen. Häufig disponieren Keimbahnmutationen von Tumorgenen zur Entwicklung mehrerer Krebsformen, aber auch zur anderweitigen Störung der Gewebedifferenzierung. Hereditäre Krebserkrankungen zeigen erhebliche Variabilität hinsichtlich des Manifestationsalters und des Krankheitsspektrums und bieten eine für die Beratung, Diagnostik, Prävention und Therapie komplexe Problematik.

Für einige dieser hereditären Krebserkrankungen existieren nach Genanalyse bereits überzeugende präventive und therapeutische Konzepte. Wegen der relativen Häufigkeit von Krebserkrankungen in der Bevölkerung beweisen mehrere betroffene Mitglieder in einer Familie jedoch nicht unbedingt eine vererbte Tumordisposition. Deshalb sollen vorher Aussagen der genetischen Diagnostik wohl überlegt sein, der mögliche Nutzen muss gegenüber denkbaren Nachteilen für den Untersuchten und seine Familie abgewogen werden. Jede der verschiedenen Krebsdis- positionen erfordert sehr spezielle Vorsorgeuntersuchungen und Früherkennungsmaßnahmen. Die Grundsätze von genetischer Diagnostik, Beratung und Prävention sind bei den verschiedenen Krebsdispositionen jedoch weitgehend gleichartig.

Die prädiktive Diagnostik von $\mathrm{Tu}$ morkrankheiten wirft bislang unbekannte ethische Probleme auf. Da es um Erkrankungsrisiken bislang gesunder Personen geht, muss an die neuen diagnostischen Möglichkeiten mit besonderer Umsicht herangegangen werden. Leitlinien zur Qualitätssicherung sind erforderlich - sowohl für die Beratung der Patienten und ihrer Angehörigen als auch für die Laboruntersuchungen. Beratung und Betreuung von Patienten aus Familien mit genetischer Krebsdisposition sind nur durch ein interdisziplinäres Vorgehen gewährleistet. Erst nach Erörterung eines adäquaten Betreuungskonzeptes kann die Eingrenzung des individuellen Risikos durch eine molekulargenetische Diagnostik erwogen werden, dies muss gezielte Vorsorgeuntersuchungen und ein Behandlungskonzept zur Folge haben.

Es hängt von der Art der Krebserkrankung ab, welche Früherkennungsmaßnahmen und Vorsorgeuntersuchungen sinnvoll sind, ab welchem Alter sie durchgeführt werden sollten, welche therapeutischen Möglichkeiten zur Verfügung stehen und wie effektiv diese sind. Prädiktive genetische Diagnostik kann andererseits vielfach erreichen, dass diejenigen, die die Keimbahnmutation nicht geerbt haben, aus dem intensiveren Vorsorgeprogramm entlassen werden können. Die Effizienz 
der Vorsorgeuntersuchung sowie eine eventuelle präventive Behandlung bedürfen der wissenschaftlichen Kontrolle und berühren Kernbereiche der Privatsphäre des Menschen.

Um Diskriminierung vorzubeugen, ist ein Patient oder eine Person mit erhöhtem Krebsrisiko vor dieser genetischen Untersuchung umfassend über die Bedeutung und Tragweite zu informieren und zu beraten. Die Freiwilligkeit jeder genetischen Untersuchung und die Vertraulichkeit der erhobenen genetischen Daten müssen gewährleistet sein. Gesunden Personen ohne auffällige Familienanamnese sollten Untersuchungen auf Krebsdispositionsgene nicht angeboten werden.

\section{Rolle des Pathologen}

Trotz der zahlreichen Fortschritte im Verständnis der Molekulargenetik von familiären Tumorsyndromen in den letzten 10 Jahren sind wir in der täglichen Praxis noch weit davon entfernt, alle Patienten mit solchen Erkrankungen zu identifizieren. Aus diesem Grund haben sowohl der Pathologe als auch der Kliniker die Verantwortung, Patienten zu erkennen, die einer humangenetischen Beratung und einer eventuellen Gendiagnostik in spezialisierten Zentren zugeführt werden sollen. Nur so werden wir auch gesicherte Erkenntnisse über die Häufigkeit dieser Syndrome in Deutschland gewinnen. Ein sehr gutes Beispiel, dass durch flächendeckende Erfassung von hereditären Tumorsyndromen weitreichende Erkenntnisse über die Häufigkeit und die Relationen von molekularen Veränderungen zum klinischen Phänotyp gewonnen werden können, ist das durch die Deutsche Krebshilfe initiierte Verbundprojekt „Familiärer Darmkrebs".

Dem Pathologen kommt dabei eine ganz zentrale Rolle zu. Häufig ist er es, der die Kliniker auf die Möglichkeit des Vorliegens einer vererbten Tumorerkrankung hinweist. Dies liegt vor allem an der zentralen Stellung des Pathologen im Netzwerk der medizinischen Betreuung und daran, dass der Pathologe über ein breites Wissen in der Diagnostik von Erkrankungen aller Fachdisziplinen und Organsysteme verfügen muss. Viele der Tumoren, die auf der Grundlage von fa- miliären Tumorerkrankungen mit Keimbahnmutationen in Tumorsuppressorgenen und Onkogenen entstehen, sind sowohl auf molekulargenetischer Ebene als auch histopathologisch in den letzten Jahren gut charakterisiert worden. Es wurde klar, dass Tumoren mit hereditärer Komponente spezifische morphologische Eigenschaften aufweisen können, die der Pathologe erkennen kann. Deshalb ist es heute wichtig, dass der diagnostisch tätige Pathologe diese histopathologisch erkennbaren morphologischen Tumorentitäten diagnostiziert und richtig einordnet. Zusätzlich wird oft erst bei der pathologischen Befundung deutlich, dass ein $\mathrm{Pa}$ tient eine Häufung bestimmter Tumoren aufweist, die ebenfalls wichtige Hinweise auf das Vorliegen eines bestimmten familiären Tumorsyndroms geben können.

Im vorliegenden Themenheft „Familiäre Tumorerkrankungen“ werden ausgewählte morphologische Tumorentitäten dargestellt, die sicherlich in der täglichen Praxis heute noch nicht sehr häufig als solche diagnostiziert werden. Dabei können nicht alle hereditären Tumorsyndrome umfassend abgehandelt werden. Daher konzentrieren wir uns besonders auf gastrointestinale Tumoren, Nierenzellkarzinome, Mammakarzinome, Sarkome, Hirntumoren und endokrine Tumoren. Bewusst steht dabei die Histopathologie dieser Tumoren im Zentrum.

Das Ziel dieses Themenhefts ist einerseits die Darstellung der morphologischen Eigenschaften relativ häufiger Tumoren, die als Warnsignal (Indextumor) für das Vorliegen einer familiären Tumorerkrankung genutzt werden können. Hier sind insbesondere gutartige bzw. bösartige Hauttumoren zu nennen, die einen ersten Hinweis für das Vorliegen eines hereditären Tumorsyndroms geben können und deswegen in einer gesonderten Arbeit behandelt werden. Andererseits werden auch seltene Tumoren dargestellt, die aber hochspezifisch für bestimmte Syndrome sind. Schließlich werden Patienten mit einer richtungsweisenden Kombination verschiedener Tumoren besprochen.

Wir hoffen, dass wir mit dem vorliegenden Themenheft dem diagnostisch tätigen Pathologen einen Leitfaden in die Hand geben können, mit dem er in der täglichen Diagnostik mögliche hereditäre
Tumorsyndrome erkennen und für den jeweiligen Patienten dann eine weitere Abklärung mit initiieren kann.
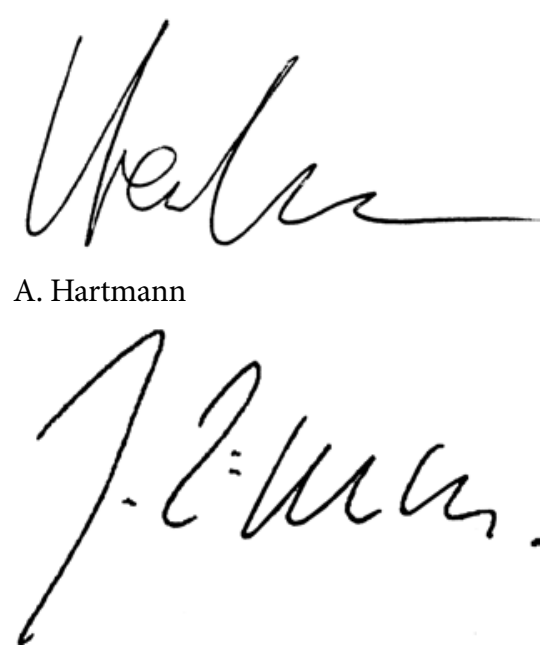

J. Rüschoff

\section{Korrespondenzadresse}

Prof. Dr. A. Hartmann

Institut für Pathologie, Universität Erlangen

Krankenhausstr. 12, 91054 Erlangen

arndt.hartmann@uk-erlangen.de 